\title{
Editorials
}

\section{How do women cope with family violence? Moving ahead in our understanding of international issues}

Violence against women and girls is considered a major health and human rights issue. In 1993, the United Nations General Assembly adopted a declaration on the elimination of violence against women. Yet, although family violence has been recognised as a public health problem for almost a decade, ${ }^{1}$ the research on family violence has produced results that are difficult to integrate either conceptually or empirically.

The World Health Organisation's Violence and Injury Prevention Department plans to publish a report on Violence and Injury Prevention by the end of the year 2001. A chapter of the report will focus on violence against women by intimate partners. A description of the magnitude of the problem will include an analysis of women's response to abuse, the consequences of abuse for health and well being, as well as preventive and policy issues. The authors of the report will also tackle the issue of data comparability when investigating domestic violence in different countries. ${ }^{2}$

The article by Ellsberg et al in this issue (see page 547) makes an important contribution to our understanding of women's response to violence by analysing the situation in Nicaragua and comparing it with what is currently known in other countries. Research has shown that violence against women can take different forms and there may be distinctive manifestations of gender violence and ways of coping with it in different cultures or regions. ${ }^{3}$ Ellsberg et al however, find that qualitative studies conducted in North America, Europe and other parts of the world show that "the barriers to leaving abusive relationships are surprisingly similar."

Interestingly, the authors found that in Nicaragua there was no association between poverty, education, number of children or marital status and any of the three coping mechanisms investigated. However, the women who separated sooner from an abusive partner were those who were under 25 years of age, had more education, and did not have a history of violence in their families.

Although women who experienced severe abuse were more likely to leave the relationship or seek help, none of the rural women in the study sought help through formal channels despite suffering severe violence. A key contribution of the study is to uncover the need for more interventions to help women to deal with violence.

While most countries formally recognise that gender violence is a crime very little is done in the way of implementing active preventive and early intervention measures. We are still far from broadening the perception that gender violence is not only an individual and family problem, but a society problem as well. The study by Ellsberg et al shows that community and family support can help women to overcome domestic violence.

The increasing number of rigorous studies on the topic, escalating statistics and tragic stories that become highline news do not seem sufficient to change society's level of tolerance and misconceptions about what has been a shameful societal problem for centuries. This is certainly a case where researchers should work hand by hand with policy makers - and a first and necessary step may be to focus on educating health professionals.

Most practising physicians never receive formal education on family violence. A recent survey of US medical schools showed that despite an increase in the number of medical schools reporting having a curriculum in family violence, since 1987, there have been no changes in the total instruction time or in the curriculum during clinical training. ${ }^{4}$

Furthermore, nurses are also an important collective to mobilise in the prevention, and recognition of family violence. As Clapp notes ${ }^{5}$ stopping domestic violence requires a community response, including close collaboration between health providers at all levels and social service agencies.

Apart from specific recommendations, a pivotal factor for success is true commitment by those who hold the political power in their hands. Joining forces and denouncing those governments that do not take the necessary steps to enforce the United Nations declaration of human rights to stop violence against women should be the responsibility of all health providers or researchers. Family violence should no longer be understood as a private issue but as an international one.

EUNICE RODRIGUEZ

Department of Policy Analysis and Management, N140 MVR Hall, Cornell University, Ithaca, NY 14853-4401, USA (er23@cornell.edu)

1 US Department of Health and Human Services Public Health Service. Healthy people 2000: national health promotion and disease prevention objectives. Healthy people 2000: national health promotion and disease

2 For additional information see http://www.who.int/

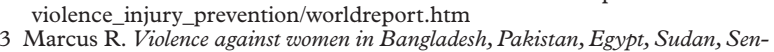
Marcus R. Violence against women in Bangladesh, Pakistan, Egypt, Sudan, Sen-
egal and Yemen. Bridge report no 10. Brighton: Institute of Development Studies, 1993.

4 Alpert EJ, Tonkin AE, Seeherman AM, et al. Family violence curricula in US medical schools. Am f Prev Med. 1998;4:273-82.

5 Clapp L. Ending domestic violence is everyone's responsibility: an integrated approach to domestic violence treatment. Nurs Clin North Am 2000;35:481-8. 